\title{
The Use of Social Media in Endourology: An Analysis of the 2013 World Congress of Endourology Meeting
}

\author{
Noah E. Canvasser, MD, Christina Ramo, BS, Todd M. Morgan, MD, Kai Zheng, PhD, \\ Brent K. Hollenbeck, MD, MS, and Khurshid R. Ghani, MBChB, MS, FRCS (Urol) ${ }^{1}$
}

\begin{abstract}
Objective: To examine the use of social media within Endourology by reporting on its utilization during the 2013 World Congress of Endourology (WCE) annual meeting.

Materials and Methods: Two social media platforms were analyzed for this study: Twitter (San Francisco, CA) and LinkedIn (Mountain View, CA). For Twitter, a third-party analysis service (Tweetreach) was used to quantitatively analyze all tweets with the hashtags \#WCE2013 and \#WCE13 during a 7-day period surrounding the WCE. Two reviewers independently classified tweet content using a predefined Twitter-specific classification system. Tweet sentiment was determined using sentiment analysis software (Semantria, Inc., Amherst, MA). Finally, the penetration of Twitter and LinkedIn within the WCE faculty was assessed by means of a manual search.

Results: During the study period, 335 tweets had the hashtag \#WCE2013 or \#WCE13. Content originated from 68 users resulting in a mean of 47 tweets/day and 4.9 tweets/contributor. Conference-related tweets had a reach of 38,141 unique Twitter accounts and an online exposure of 188,629 impressions. Physicians generated the majority of the content (63\%), of which $55.8 \%$ were not attending the meeting. More tweets were informative $(56.7 \%)$ versus uninformative $(43.3 \%)$, and $17.9 \%$ had links to an external web citation. The mean sentiment score was 0.13 (range -0.90 to 1.80 ); $13.1 \%, 57.0 \%$, and $29.9 \%$ of tweets were negative, neutral, and positive in sentiment, respectively. Of 302 WCE meeting faculty, $150(49.7 \%)$ had registered LinkedIn accounts while only $52(17.2 \%)$ had Twitter accounts, and only $19.2 \%$ tweeted during the meeting.

Conclusions: Despite a relatively low number of Twitter users, tweeting about the WCE meeting dramatically increased its online exposure with dissemination of content that was mostly informative including engagement with physicians not attending the conference. While half of faculty at WCE 2013 had LinkedIn accounts, their social media footprint in Twitter was limited.
\end{abstract}

\section{Introduction}

O ocial Media IS THE TERM USED to describe Internet-based $\checkmark$ tools that allow for the creation and exchange of usergenerated content. Social media applications have entered the landscape in many fields; in healthcare it has been used for peer-to-peer and clinician-to-patient communication, to promote institutional branding, and improve the speed of interaction across different stakeholders. ${ }^{1}$ Advances in mobile-phone technology have led to highly interactive platforms on smartphones that allow individuals and communities to create, share, discuss, and modify user-generated content at any time.

Two popular social media platforms used by physicians are Twitter ${ }^{2}$ and LinkedIn. ${ }^{3}$ Twitter is a "microblog" that has become the preeminent social media tool for information dissemination, social networking, and real-time communication. ${ }^{4}$ In March 2013, Twitter had over 200 million active users sending 400 million tweets per day. ${ }^{5}$ LinkedIn is a professional networking website that is focused on relationships related to one's professional career. As of 2014, LinkedIn has over 250 million users in more than 200 countries. $^{6}$

Within urology, the relevance of these social media tools has been the subject of commentary ${ }^{7}$ and evaluation by professional associations such as the American Urological Association (AUA). ${ }^{8}$ More recently, there has been great interest in their role in education and information dissemination, especially during medical conferences. ${ }^{9-12}$ The aim of this study was to determine the use of social media within Endourology

\footnotetext{
${ }^{1}$ Department of Urology, University of Michigan, Ann Arbor, Michigan.

${ }^{2}$ Department of Health Management and Policy, School of Information, University of Michigan, Ann Arbor, Michigan.
} 
by evaluating its utilization during the 2013 World Congress of Endourology (WCE) annual meeting. We set out to determine analytic metrics of Twitter use during the meeting as well as understand the content of tweets created. Finally, to gauge the impact of social media within opinion leaders of Endourology, we examined the penetration of Twitter and LinkedIn among the faculty of the WCE meeting.

\section{Materials and Methods}

The WCE annual meeting in 2013 was held in New Orleans, United States, from October 22 to 26. The conference, hosted by the Endourology society and open to both members and nonmembers, is the largest international meeting on minimally invasive urologic surgery. In 2013, there were over 1900 attendees and 302 faculties at the meeting. ${ }^{13}$

\section{Twitter}

The Appendix Table A1 provides a glossary of Twitter terminology. During the meeting, Twitter users discussing the WCE meeting used the hashtags \#WCE2013 or \#WCE13. Following completion of the meeting, all tweets with these hashtags during the dates of the meeting, including the day before and after (October 21-27, 2013), were obtained from a third-party Twitter analysis service (Tweetreach, San Francisco, CA). This service analyzes tweets to provide metrics of use, including reach (the total number of unique Twitter users who received a tweet about the search term) and exposure (total number of times tweets about the search term were delivered to Twitter streams).

\section{Twitter: quantitative analysis}

All captured tweets along with the associated Twitter accounts were reviewed and categorized by two reviewers. Any discrepancy among reviewers was managed by the senior reviewer making the final decision. Through a manual search, Twitter users were identified by registered country, and placed into one of eight categories: physician-urologist, physiciannonurologist, medical journal, professional association, media/ news organization, department/institution, or industry/commercial venture. Individual users were also identified as either attending or not attending the meeting by content analysis of tweets.

\section{Twitter: qualitative analysis}

Tweets were analyzed for content, citation, and sentiment. Content was categorized as either informative or uninformative of any aspect of urologic diseases using a previously established classification system ${ }^{14}$ that has already been used to study twitter use during a medical conference. ${ }^{10}$ Uninformative tweets were subdivided into (1) advertisement, (2) status update, (3) query, (4) direct message, (5) opinion, or (6) other. Tweets were also analyzed to determine the presence of Web citations (external, internal, or none) based on a predefined Twitter-specific classification system. ${ }^{15}$ Tweets with internal citations are commonly referred to as re-tweets (Appendix Table A1) while external citations contain shortened uniform resource locators (URLs) to third-party websites. Each tweet was also evaluated for sentiment ${ }^{16}$ using a Twitter-specific sentiment software tool (Semantria, Inc., Amherst, MA). The positive, neutral, or negative feeling bound to the text for each tweet was processed to provide a numeric score ranging from -2 and +2 to indicate negative, neutral, or positive sentiment.

\section{Social media activity of faculty at WCE meeting}

Meeting faculty were identified using the conference program, and a manual search was performed to determine how many faculty members had user accounts on Twitter and LinkedIn. Those with Twitter accounts were checked to see whether tweets related to the WCE meeting were generated during the study period.

\section{Results}

\section{Quantitative analysis}

In total there were 68 Twitter users creating 335 tweets with the hashtags \#WCE2013 or \#WCE13 during the 7-day study period. \#WCE2013 was the most popular hashtag, comprising $296(88.4 \%)$ of the tweets. Users originated from 14 different countries, the largest contingent being from the United States (63.2\%). Table 1 provides the descriptive characteristics of Twitter users. Of the 43 physicians tweeting during the meeting, $24(55.8 \%)$ were not actually attending the meeting.

Of the 335 tweets, 217 were original tweets, 73 were retweets, and 45 were direct replies to tweets. Figure 1 provides an example of a tweet generated during the meeting. Apart from one, all tweets were related to the WCE meeting; this tweet was in reference to a nonmedical meeting. Figure 2 demonstrates the daily Twitter activity during the study period. The mean number of tweets per day was 47 (range $=1-92$ ) and the mean number of tweets per contributor was 4.9 (range $=1-55$ ). Activity peaked on the second day of the meeting with 92 tweets. The total reach of tweets during the

Table 1. Descriptive Characteristics OF TWITTER USERS DURING WORLD Congress of Endourology 2013 Meeting

Characteristics of Twitter

contributors $(\mathrm{n}=68)$

n

Physician: urologist

Physician: nonurologist

Journal

Professional association

Media/news organization

Department/Institution

Industry

42 1

Countries

United States

United Kingdom

Canada

Australia

El Salvador

Brazil

Columbia

Egypt

France

Italy

Panama

Indonesia

Turkey

Venezuela 
FIG. 1. Example of a tweet created by Dr. Jason Lee from Toronto at the time of the World Congress of Endourology (WCE) 2013 annual meeting. The tweet, as visualized on the Twitter platform, shows the name of the author (in bold), a profile photo, their twitter account name (also known as "Twitter handle"), and the date of the tweet. The hashtag \#wce2013 indicates that the tweet concerns the WCE meeting. The commands in the bottom right allow viewers to (1) comment by pressing "reply"; (2) "retweet" the post, which would further amplify the message to other users; and (3) express interest in the tweet by pressing "favorite."

study period was 38,141 , meaning this was the total number of individual unique Twitter accounts that received tweets with the hashtag \#WCE2013 or \#WCE13. The overall exposure for the entire meeting was 188,629 impressions, meaning the total number of times tweets were delivered to timelines including repeats. Table 2 lists the top 10 contributors on Twitter based on total exposure.

\section{Qualitative analysis}

Figure 3 displays the breakdown of tweets based on content. Most tweets were informative (190; 56.7\%). Of this group, 75 tweets $(39.5 \%)$ contained content delivered during the meeting, such as abstracts or plenary items. The status update was the most common uninformative tweet (61; $42.1 \%$ of uninformative tweets). Table 3 provides examples of informative and uninformative tweets. External web citations with URLs were seen in 60 (17.9\%) tweets. Informative tweets had a significantly higher external citation count than uninformative tweets: 43/190 (48\%) versus 17/ $145(12 \%)$, respectively ( $p=0.014$; Fisher's exact test). Pictures were attached to tweets in 111/335 (33.1\%) instances. Sentiment analysis of tweets demonstrated a range of sentiment during the meeting (Fig. 4). The mean sentiment score was 0.13 (range -0.90 to 1.80 ). In total, few tweets were negative in sentiment; $29.9 \%, 57.0 \%$, and $13.1 \%$ were positive, neutral, and negative, respectively.

\section{Social media presence among WCE faculty}

There were 302 teaching and speaking faculty at the WCE 2013. Of these, the number with registered user accounts on Twitter was $52(17.2 \%)$, of which only $10 / 52(19.2 \%)$ tweeted during the study period. In total, the number of faculty tweeting at the meeting was very low (3.3\%). In contrast, the number of faculty members on LinkedIn was much higher $(49.7 \%)$.

\section{Discussion}

In this study, we examined the utilization of social media during urology's largest annual Endourology meeting. In particular, we evaluated metrics of Twitter use, including content and sentiment of tweets during the meeting. Although the number of Twitter users generating content was small, information regarding the conference was disseminated to a much larger online audience. While there were a variety of content generators, physicians created the majority of content and more than half of them engaged in discussion despite not attending the meeting. Few tweets were negative in sentiment, suggesting that the meeting was well received on social media or that users are unlikely to put critical content surrounding the meeting on Twitter. However, we found that only a few WCE faculty members had Twitter accounts and only a small proportion tweeted during the meeting. In

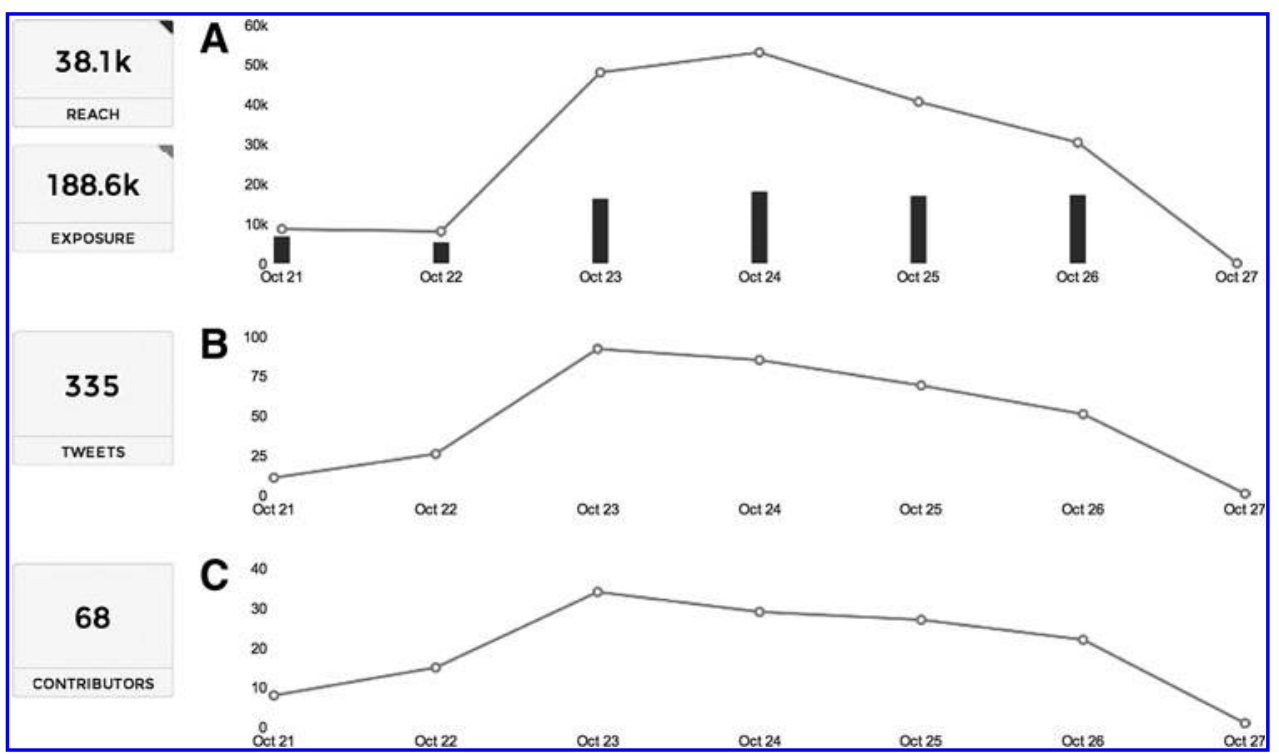

FIG. 2. Twitter use during the 2013 WCE; Data from Tweetreach. (A) The reach (bar) and exposure (line) per day for tweets using hashtags \#WCE2013 and \#WCE13 per day. (B) The number of tweets per day with hashtags \#WCE2013 and \#WCE13. (C) The number of contributors tweeting per day. 
Table 2. Top 10 Twitter Contributors at World Congress of Endourology 2013 MeEting

\begin{tabular}{lccr}
\hline Username & $\begin{array}{c}\text { Number } \\
\text { of tweets }\end{array}$ & $\begin{array}{c}\text { Number } \\
\text { of re-tweets }\end{array}$ & $\begin{array}{c}\text { Total } \\
\text { exposure }\end{array}$ \\
\hline @urotoday & 19 & 2 & 44,400 \\
@Tdave & 55 & 4 & 26,347 \\
@ healthhashtags & 9 & 2 & 17,643 \\
@MedThriller & 8 & 2 & 16,974 \\
@peepeeDoctor & 55 & 8 & 16,840 \\
@UrologyMatch & 7 & 0 & 15,493 \\
@benchallacombe & 13 & 4 & 13,039 \\
@ cookuro & 8 & 2 & 11,922 \\
@qdtrinh & 12 & 3 & 7740 \\
@WordSatSpangalo & 2 & 0 & 6787 \\
\hline
\end{tabular}

contrast, nearly half of all meeting faculties had an account on the social media platform LinkedIn.

Social media can be a valuable tool for urologists, and Twitter can serve a variety of functions. It may be a platform for promoting education, advocacy, networking and socializing, crowd-sourcing, advertising as well as dissemination of news, emerging research, and information from conferences. ${ }^{17}$ In a survey of AUA members, $74 \%$ of respondents had a social media account, with the most popular being Facebook (93\%) followed by LinkedIn (46\%) and Twitter $(36 \%) .{ }^{8}$ More recently, Twitter has been used to conduct an online urology journal club (@iurojc) with over 1000 users following or contributing to scientific comment worldwide. ${ }^{18}$ This activity demonstrated the potential of social media for facilitating international communication that had not been possible before.

The use of Twitter at medical conferences has been the subject of recent studies. ${ }^{3,9-12}$ An analysis of the 2013 Academic Surgical Congress revealed that 58 users provided 435 tweets during this meeting. ${ }^{9}$ Similar to our findings, the majority of participants tweeting were not attending the conference, illustrating the unique ability of Twitter to allow engagement with those not physically present. Consequently, its use at meetings is growing. In a study of the American

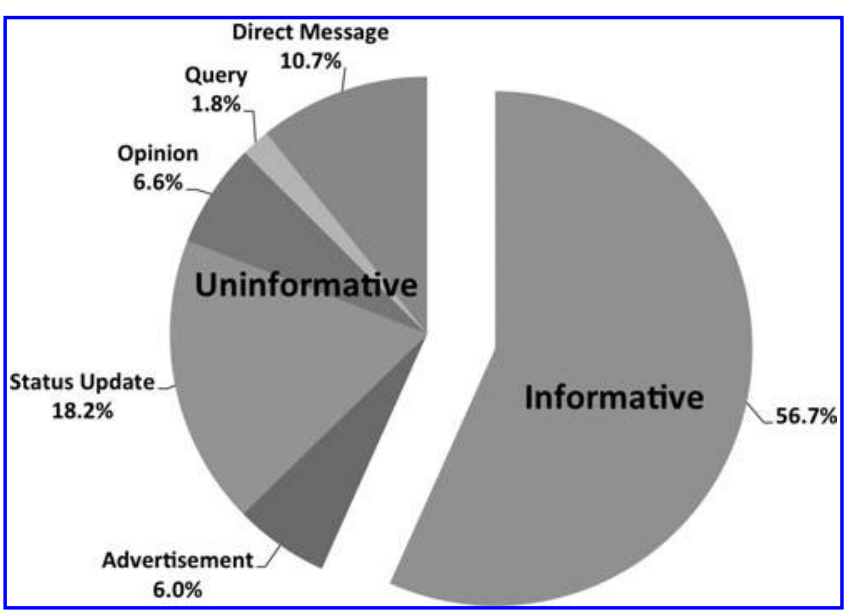

FIG. 3. Classification of the content of tweets with hashtags \#WCE2013 and \#WCE13.
TABle 3. EXAmples of Informative AND UNINFORMATIVE TWEETS

\begin{tabular}{|c|c|}
\hline Content & Tweet \\
\hline Informative & $\begin{array}{l}\text { @ peepeeDoctor \#wce2013 If doing } \\
\text { semi-rigid, y not basket then laser } \\
\text { stone. No flow limitations w/1.5F } \\
\text { basket + laser in semirigid \#cheaper }\end{array}$ \\
\hline $\begin{array}{l}\text { Uninformative- } \\
\text { advertisement }\end{array}$ & $\begin{array}{l}\text { RT @qdtrinh: Shout out to \#WCE2013 } \\
\text { attendees_follow @UMichUrology }\end{array}$ \\
\hline $\begin{array}{l}\text { Uninformative- } \\
\text { status update }\end{array}$ & $\begin{array}{l}\text { RT @allaf_mo: Ted Deweese } \\
\text { \#wce2013 keynote address re } \\
\text { Nanomedicine. "I am a urologist } \\
\text { at heart" "I grew up in the } \\
\text { @brady_urology", @alan_pa }\end{array}$ \\
\hline $\begin{array}{l}\text { Uninformative- } \\
\text { opinion }\end{array}$ & $\begin{array}{l}\text { @ Tdave \#wce } 2013 \text { If she has a ureter, } \\
\text { I would perform ureteroscopy! Great } \\
\text { panel, Tim. }\end{array}$ \\
\hline $\begin{array}{l}\text { Uninformative- } \\
\text { query }\end{array}$ & $\begin{array}{l}\text { Hey \#wce } 2013 \text {, where are the reports } \\
\text { on @ DrHLN poster presentation } \\
\text { today? }\end{array}$ \\
\hline $\begin{array}{l}\text { Uninformative- } \\
\text { direct message }\end{array}$ & $\begin{array}{l}\text { Next year \#wce } 2013 \\
\text { "@ @JYLeeUroSMH: @DrRKSingal } \\
\text { @Tdave wish u were here too } \\
\text { Rajiv!”" }\end{array}$ \\
\hline
\end{tabular}

$\mathrm{RT}=$ re-tweet WCE $=$ World Congress of Endourology.

Society of Clinical Oncology 2010 and 2011 annual meetings, tweets and impressions increased significantly from 1 year to the next. ${ }^{11}$ As in our study, the proportion of attendees actively tweeting was relatively small, only 34 physicians compared with over 30,000 attendees. While most studies have showed a trend toward small groups of attendees composing tweets, ${ }^{3,10}$ some meetings have been characterized by very large numbers of attendees using Twitter during the meeting, suggesting that social media penetration may display variability according to specialty. ${ }^{12}$

So far, an analysis of Twitter at urological meetings has been limited to two studies. A brief study of the 2013 AUA meeting revealed a total of 5058 tweets from 644 contributors

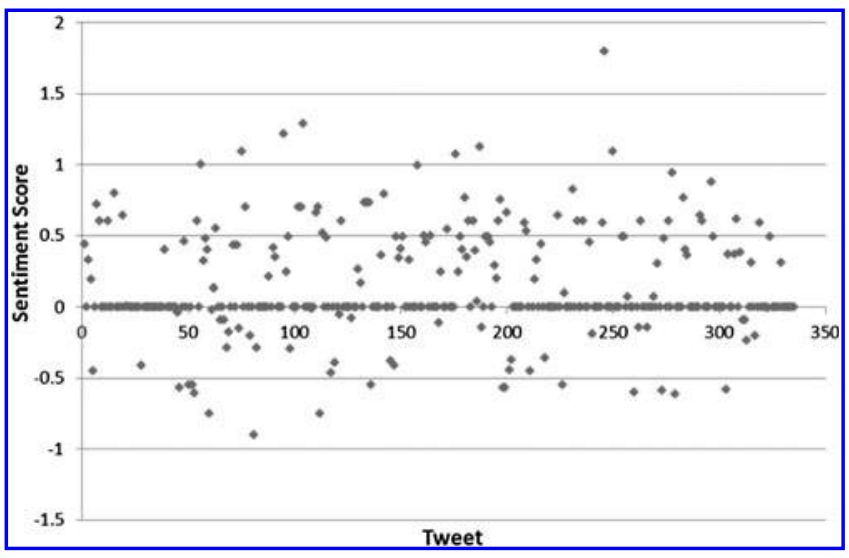

FIG. 4. Sentiment analysis of tweets with hashtags \#WCE2013 and \#WCE13 posted during study period. Each tweet was given a numerical sentiment score ranging from -2 (negative) to +2 (positive). 
resulting in over 9 million impressions. ${ }^{8}$ Matta et al. compared the Canadian and AUA meetings of 2012-2013, and found an increase in the frequency of tweets as well as the frequency of informative tweets. The majority of the tweets were related to oncology (69\%) followed by endourology and stone disease $(10 \%) .{ }^{19}$ Our study provides the first analysis of Twitter during an endourology meeting.

We also assessed the degree to which key opinion leaders in endourology, such as faculty of the WCE meeting, had a footprint in social media. LinkedIn was much more popular with faculty than Twitter. This popularity of LinkedIn over Twitter was also confirmed in a study of an emergencymedicine conference. ${ }^{3}$ While LinkedIn remains a good source for establishing professional networks, unlike Twitter it does not allow real-time interactive commentary and feedback. Twitter appears to be more popular among residents and younger physicians $($ age $<40) .{ }^{8}$ It may be that the studied faculty represents a different generation of physicians who have not yet embraced this social media platform. This may change as one of the hallmarks of Twitter is the unparalleled ability for free and open access to opinion leaders. ${ }^{18}$

Our study has a number of limitations. First, we used a retrospective method for collecting Tweets. However, it is unlikely that we failed to collect all tweets with the studied hashtags as we employed a third-party system with access to the full archive of public Twitter data. Another limitation is that we decided to study only those tweets with particular meeting-related hashtags. It is possible that we missed some tweets about the meeting that did not use these hashtags. Also, while we could easily identify whether twitter users generating content were physicians, the retroactive search for Twitter accounts of faculty members based on names can be arduous, especially if the user has a common name. We found this to be the case in four individuals. Further, we limited our assessment of professional networking platforms to LinkedIn, although there are newer platforms that are physician specific, such as Doximity (San Mateo, CA, USA). Finally, the measurement of impressions in this arena is subjected to bias. It only takes one Twitter account with a very large following to increase the amplification of a tweet and the impression count substantially. There is no way to know whether or not a particular user has actually read a Tweet delivered to their account. Not everyone who receives a tweet will read it.

Twitter's role in information dissemination during medical meetings is likely to increase in the future. Indeed, commercial enterprises have now sprung up whose sole purpose is to analyze Twitter metrics of medical meetings. ${ }^{20}$ Some associations have created "Twitter Teams" to promote the meeting hashtag during the conference to increase engagement. ${ }^{9}$ It is even becoming customary for urological conferences to promote the meeting hashtag in advance. ${ }^{21}$ Other future directions we foresee include the use of sentiment analysis to gauge feedback from attendees rather than relying on emailed surveys. Sentiment analysis is the current standard by which corporations determine the polarity of content created by consumers. It uses computational algorithms based on natural language processing techniques and is emerging as the standard for assessing attitudes within tweets. Further, the custom of announcing the best article at a conference may expand to include awards for the best tweet. As such, our study provides some information on how Twitter is, and can be used, during a urology meeting.

\section{Conclusions}

The use of Twitter at the 2013 WCE meeting allowed for real-time amplification of informative updates to an international audience including many physicians who were not physically present at the meeting. While the number of congress faculty with Twitter accounts was low compared with those with LinkedIn, we expect increased adoption of Twitter by all stakeholders at future urologic conferences.

\section{Acknowledgment}

The authors thank Professor John Wei, Department of Urology, University of Michigan, for reading the article and his advice on social media in urology.

\section{Disclosure Statement}

No competing financial interests exist.

\section{References}

1. Grajales FJ, Sheps S, Ho K, Novak-Lauscher H, Eysenbach G. Social media: A review and tutorial of applications in medicine and health care. J Med Internet Res 2014;16:e13.

2. Chretien KC, Azar J, Kind T. Physicians on Twitter. JAMA 2011;305:566-568.

3. Neill A, Cronin JJ, Brannigan D, O'Sullivan R, Cadogan M. The impact of social media on a major international emergency medicine conference. Emerg Med J 2014;31:401-404.

4. Bilton N. Hatching Twitter: A True Story of Money, Power, Friendship, and Betrayal. New York: Penguin Book Group, 2013, p. 302.

5. Tsukayama H. Twitter turns 7: Users send over 400 million tweets per day: The Washington Post; 2013 [cited 2014 March 31]. Available from: www.washingtonpost.com/ business/technology/twitter-turns-7-users-send-over-400million-tweets-per-day/2013/03/21/2925ef60-9222-11e2bdea-e32ad90da239_story.html

6. Fiegerman S. LinkedIn Tops 250 Million Members: Mashable; 2013 [cited 2014 March 31]. Available from: http://mashable.com/2013/10/29/linkedin-q3-earnings/

7. Trinh Q-D. Why I care about social media-and why you should too. BJU Int 2013;112:1-2.

8. Loeb S, Bayne CE, Frey C, et al. Use of social media in urology: Data from the American Urological Association. BJU Int 2014;113:993-998.

9. Logghe H, Maa J, Schwartz J. Twitter usage at Clinical Congress rises markedly over two years. Bull Am Coll Surg 2013;98:22-24.

10. Desai T, Shariff A, Shariff A, et al. Tweeting the meeting: An in-depth analysis of Twitter activity at Kidney Week 2011. PLoS One 2012;7:e40253.

11. Chaudhry A, Glode LM, Gillman M, Miller RS. Trends in twitter use by physicians at the american society of clinical oncology annual meeting, 2010 and 2011. J Oncol Pract 2012; 8:173-178.

12. Hawkins CM, Duszak R, Rawson JV. Social media in radiology: Early trends in Twitter microblogging at radiology's largest international meeting. J Am Coll Radiol 2014;11:387-390.

13. Ghani KR. Dusting vs. Fragmentation and other highlights from WCE 2013: BJUI; 2013 [cited 2014 March 31]. Available from: www.bjuinternational.com/bjui-blog/worldcongress-of-endourology

14. Dann S. Twitter content classification. First Monday 2010; 15:1-12. 
15. Weller K, Puschmann C. Twitter for Scientific Communication: How Can Citations/References be Identified and Measured? Koblenz, Germany, 2011. Available from: http:// journal.webscience.org/500/1/153_paper.pdf

16. Agarwal A, Xie B, Vovsha I, Rambow O, Passonneau R. Sentiment analysis of Twitter data. Proceedings of the Workshop on Languages in Social Media. Portland, Oregon: Association for Computational Linguistics, 2011, pp. 30-38.

17. Loeb S. Twitter in Urology; 2013 [cited 2014 March 31]. Available from: www.urologymatch.com/node/4253

18. Thangasamy IA, Leveridge M, Davies BJ, Finelli A, Stork B, Woo HH. International Urology Journal Club via Twitter: 12-Month Experience. Eur Urol 2014;66:112-117.

19. Matta R, Doiron C, Leveridge MJ. The dramatic rise of social media in urology: Trends in Twitter Use at the American and Canadian Urological Association Annual Meetings in 2012 and 2013. J Urol 2014;192:494-498.

20. Symplur. Connecting the $\overline{\text { dots }}$ in healthcare social media [cited 2014 March 31]. Available from: www.symplur .com
21. European Association of Urology. Seven reasons why you should be on Twitter for \#EAU14 2014 [cited 2014 March 31]. Available from: www.eaustockholm2014.org/?id= $65 \&$ aid $=803$

Address correspondence to: Khurshid R. Ghani, MBChB, MS, FRCS (Urol) Department of Urology University of Michigan Building 16, First Floor 2800 Plymouth Road Ann Arbor, MI 48109

E-mail:kghani@med.umich.edu

$\begin{aligned} & \text { Abbreviations Used } \\ \mathrm{AUA}= & \text { American Urological Association } \\ \mathrm{URL}=\text { uniform resource locator } & \mathrm{W} \text { W }=\text { World Congress of Endourology }\end{aligned}$

\section{Appendix Table A1. Glossary of Twitter Terms}

\begin{tabular}{|c|c|}
\hline Tweet & A user-generated piece of text limited to a maximum of 140 characters. \\
\hline Re-tweet & $\begin{array}{l}\text { Re-posting of a tweet created by another user, which can then be seen by the user's followers. Signified } \\
\text { with prefix RT. }\end{array}$ \\
\hline Hashtag & The symbol \# used as a categorization method by marking key words or topics in a tweet. \\
\hline $\begin{array}{l}\text { Followers/ } \\
\text { Following }\end{array}$ & $\begin{array}{l}\text { Each user can "follow" what another user posts and be "followed" by other users. Every follower } \\
\text { automatically receives the tweets of the user they are following. }\end{array}$ \\
\hline @ symbol & $\begin{array}{l}\text { Each user creates a username that is prefixed with this symbol. If used in a tweet, then that unique user } \\
\text { will receive the Tweet regardless of whether they are followed or not by the content generator }\end{array}$ \\
\hline Tweeting & To compose messages using Twitter \\
\hline Favorite & The term used to bookmark tweets that one may like or endorse \\
\hline
\end{tabular}

$\mathrm{RT}=$ re-tweet. 


\section{This article has been cited by:}

1. Ali H. Aldoukhi, Khurshid R. Ghani. 2017. Editorial Comment. Urology 108, 16. [Crossref]

2. Nicholas J. Farber, Christopher J. Koprowski, Parth K. Modi, Wei Wang, Justin M. Dubin, Young Suk Kwon, Sammy E. Elsamra. 2017. Twitter Use among Academic Urology Programs. Urology Practice 4:3, 269-274. [Crossref]

3. Ross J. S. Calopedos, Cindy Garcia, Prem Rashid, Declan G. Murphy, Nathan Lawrentschuk, Henry H. Woo. 2017. Citation indices for social media articles in urology. BJU International 119, 47-52. [Crossref]

4. Deanna J. Attai, Diane M. Radford, Michael S. Cowher. 2016. Tweeting the Meeting: Twitter Use at The American Society of Breast Surgeons Annual Meeting 2013-2016. Annals of Surgical Oncology 23:10, 3418-3422. [Crossref]

5. Hendrik Borgmann, Stacy Loeb, Johannes Salem, Christian Thomas, Axel Haferkamp, Declan G. Murphy, Igor Tsaur. 2016. Activity, content, contributors, and influencers of the twitter discussion on urologic oncology. Urologic Oncology: Seminars and Original Investigations 34:9, 377-383. [Crossref]

6. Johannes Salem, Hendrik Borgmann, Matthew Bultitude, Hans-Martin Fritsche, Axel Haferkamp, Axel Heidenreich, Arkadiusz Miernik, Andreas Neisius, Thomas Knoll, Christian Thomas, Igor Tsaur. 2016. Online Discussion on \#KidneyStones: A Longitudinal Assessment of Activity, Users and Content. PLOS ONE 11:8, e0160863. [Crossref]

7. J. Campagne, J. Galland, O. Mangin. 2016. Utilisation de TwitterMD en congrès : une première pour la médecine interne française. La Revue de Médecine Interne 37:7, 497-501. [Crossref]

8. Amanda Chung, Henry Woo. 2016. Twitter in urology and other surgical specialties at global conferences. ANZ Journal of Surgery 86:4, 224-227. [Crossref]

9. Johannes Salem, Hendrik Borgmann, Declan G. Murphy. 2016. Integrating Social Media into Urologic Health care: What Can We Learn from Other Disciplines?. Current Urology Reports 17:2. . [Crossref]

10. Alexander Kutikov, Henry H. Woo, James W. Catto. 2016. Urology Tag Ontology Project: Standardizing Social Media Communication Descriptors. European Urology 69:2, 183-185. [Crossref]

11. Michael J. Leveridge. 2016. The state and potential of social media in bladder cancer. World Journal of Urology 34:1, 57-62. [Crossref]

12. H. Borgmann, P. Paffenholz, T. Nestler, J. Salem. 2015. Benefits und Risiken der Twitter-Nutzung für Urologen. Der Urologe 54:7, 1023-1024. [Crossref]

13. Stacy Loeb, Christopher E. Bayne, Christine Frey, Benjamin J. Davies, Timothy D. Averch, Henry H. Woo, Brian Stork, Matthew R. Cooperberg, Tomas L. Griebling, Scott E. Eggener. 2015. Updated Survey of Social Media Use by Members of the American Urological Association. Urology Practice 2:3, 138-143. [Crossref] 